\title{
Levosimendan in Cardiac Surgery: Is it all Gold that Glitters?
}

\author{
Antonio Nenna, Salvatore Matteo Greco, Raffaele Barbato and Massimo Chello* \\ Department of Cardiovascular Surgery, Università Campus Bio-Medico di Roma, 00128 Rome, Italy
}

\section{Commentary}

Patients undergoing cardiac surgery are becoming older and with greater comorbidities, carrying an increased risk for perioperative complications which result in higher mortality and higher costs for the health care service [1]. One of the most demanding complications, in terms of morbidity and costs, is the low cardiac output syndrome (LCOS), which occurs in 10-15\% of patients undergoing cardiac surgery with cardiopulmonary bypass $[2,3]$. LCOS is generally more common among patients with impaired left ventricular function, and is managed with inotropic agents and, eventually, mechanical support such as intra-aortic balloon pump, extracorporeal membrane oxygenator or ventricular assist devices. Although recent advances in pharmacologic and mechanical treatments, short-term mortality risk for patients with LCOS remains up to 15 times higher compared to an uneventful post-operative course [1]. Most of the available inotropic agents have detrimental side effect or have a poor safety profile, thus exposing the patient to treatment-related risks and complications. The prevention and the effective treatment of LCOS is one of the pivotal requirement to improve outcomes in cardiac surgery.

In recent years, levosimendan is considered to be the ideal inotropic agent to support cardiac function in case of LCOS after cardiac surgery. Levosimendan is a calcium-sensitizing inotrope with a peripheral vasodilatory effect related to the ATP-sensitive potassium channel opening, and is able to increase cardiac output with minimal increase in myocardial oxygen consumption. Diluted with glucose $5 \%$ solution before infusion, levosimendan assumes a golden color and is administered in continuous infusion.

However, levosimendan has been investigated in many small clinical trials and observational studies until the recent publication of two multicenter, randomized, placebo-controlled clinical trials, which reassessed the effects and the importance of this drug $[2,3]$. The prophylactic administration among patients at high risk for postoperative LCOS was evaluated in the LEVO-CTS Trial [2], while the therapeutic effect among patients with ongoing myocardial dysfunction requiring inotropic support was investigated in the CHEETAH Trial [3].

In the LEVO-CTS Trial [2], performed in the United States and Canada from September 2014 to November 2016, 882 patients undergoing on-pump cardiac surgery were randomly assigned to receive either intravenous levosimendan or placebo. Prophylactic levosimendan did not result in a reduced rate of the composite end point of death, renal-replacement therapy, perioperative myocardial infarction, or use of a mechanical cardiac assist device. All patients had preoperative left ventricular dysfunction with preoperative left ventricular ejection fraction less than $35 \%$, and most patients underwent coronary artery bypass grafting (CABG).

The CHEETAH Trial [3], performed in Italy, Russia and Brazil, was performed from November 2009 to April 2016. Patients were randomly assigned to receive levosimendan or placebo, for up to 48 hours or until discharge from the intensive care unit (ICU), on top of standard care, and the primary outcome of the trial was 30-day mortality. Patients were included in case of perioperative cardiovascular dysfunction, such as preoperative left ventricular ejection fraction less than $25 \%$, preoperative support with an intra-aortic balloon pump, or the need for support with an intra-aortic balloon pump or high-dose inotropes to be weaned from cardiopulmonary bypass or within the first 24 hours after surgery. Therefore, unlike LEVO-CTS Trial, randomization was performed after the diagnosis of LCOS, and CHEETAH Trial investigated the therapeutic effect than the prophylactic effect of levosimendan administration. The trial was stopped for futility after 506 patients were enrolled, since levosimendan in addition to standard care did not result in lower 30-day mortality than placebo. Also, mechanical ventilation, hospital stay, and rates of hypotension or cardiac arrhythmias were not different between groups. Less than half patients underwent CABG, and considering only patients with impaired ventricular function, no beneficial effects were observed.

The other clinical trial currently investigating levosimendan is the levosimendan in Coronary Artery Revascularization (LICORN) trial, involving 340 patients with left ventricular dysfunction undergoing CABG [4]. Complete results of this trial are expected in the next year.

Considering the disagreement between the small observational studies and the recently published clinical trials, the proper evaluation of levosimendan in cardiac surgery dampened the early enthusiasms. It appears that levosimendan could quit from the scenes in the next years, but a great number of confounding variables should be considered when evaluating the results of those trials and the possible (in) efficacy of this compound.

First, levosimendan might benefit only patients with severe left ventricular dysfunction at baseline [5], but the ejection fraction cannot be considered as the unique marker of left ventricular impairment. The presence of mitral regurgitation, aortic valve regurgitation or ventricular septal defect might overestimate ejection fraction and reduce the number of patients with poor ventricular function included in those trials. Also, the diastolic dysfunction is not an inclusion criteria in those trials and recent evidences are pointing out the importance of myocardial relaxation for proper function.

Studies differ with regards to the timing of levosimendan administration, and it might be postulated that a drug started just before surgery for a chronic condition may not be effective in preventing perioperative injury.

Similarly, there is no consensus in terms of administered dose. Many observational studies report a loading dose followed by an infusion of at least $0.10 \mu \mathrm{g}$ per kilogram per minute $[5,6]$. In the LEVO-

*Corresponding authors: Massimo Chello, Department of Cardiovascular Surgery, Università Campus Bio-Medico di Roma, 00128 Rome, Italy, Tel: 3906225411140; Fax: 3906225411960; E-mail: m.chello@unicampus.it

Received April 25, 2017; Accepted May 15, 2017; Published May 18, 2017

Citation: Nenna A, Greco SM, Barbato R, Chello M (2017) Levosimendan in Cardiac Surgery: Is it all Gold that Glitters? J Cardiovasc Dis Diagn 5: 276. doi: 10.4172/2329-9517.1000276

Copyright: @ 2017 Nenna A, et al. This is an open-access article distributed under the terms of the Creative Commons Attribution License, which permits unrestricted use, distribution, and reproduction in any medium, provided the original author and source are credited. 
CTS Trial, levosimendan was given at a dose of $0.20 \mu \mathrm{g}$ per kilogram per minute for 1 hour, followed by a dose of $0.10 \mu \mathrm{g}$ per kilogram per minute for 23 hours, while in CHEETAH Study it is reported a tailored continuous infusion at a dose of $0.025 \mu \mathrm{g}$ to $0.20 \mu \mathrm{g}$ per kilogram per minute, with a mean infusion dose of $0.07 \mu \mathrm{g}$ per kilogram per minute $[2,3]$. CHEETAH Study used a more conservative approach, avoiding loading doses and high-dose infusions which could be associated more frequently with hypotension and other side effects. In the treatment of sepsis, LeoPARDS Study [7] proved that the addition of levosimendan to standard treatment was not associated with less severe organ dysfunction or lower mortality, but resulted in a higher risk of supraventricular tachyarrhythmias and hypotension at an infusion dose of $0.20 \mu \mathrm{g}$ per kilogram per minute. Therefore, the proper loading and infusion doses are not universally accepted and dedicated studies are warranted.

Also, the type of surgery should be taken into account when evaluating the effects of an inotrope. Considering that patients with ischemic heart disease or valvular heart disease have profound differences in cardiac physiology and response to pharmacologic treatments, the various effects of levosimendan may differ between patients who have ischemic left ventricular dysfunction and patients who have left ventricular dysfunction related to pressure or volume overload.

Finally, the economic counterpart of levosimendan administration should be considered, since the unitary cost of a vial is about 1000 $€-1500 €$. Many previously published studies justified the economic usefulness of levosimendan compared to other drug regimen [812 ], but a dedicate economic analysis of recent prospective trials will confirm or deny the previous results.

At present, recently published clinical trials do not support the administration of levosimendan as a prophylactic or therapeutic measure in the management of LCOS after cardiac surgery. Ongoing studies in cardiac surgery and in other fields will shed new light on this topic, to answer the question: "is it all gold that glitters?"

\section{Conflict of Interest}

None

\section{Funding Support}

None.

\section{Acknowledgements}

None.

\section{References}

1. Mehta RH, Grab JD, O'Brien SM, Glower DD, Haanet CK et al. (2008) Clinical characteristics and in-hospital outcomes of patients with cardiogenic shock undergoing coronary artery bypass surgery: insights from the Society of Thoracic Surgeons National Cardiac Database. Circulation 117: 876-885.

2. Mehta RH, Leimberger JD, van Diepen S, Meza J, Wang A, et al. (2017) Levosimendan in patients with left ventricular dysfunction undergoing cardiac surgery. N Engl J Med 376

3. Landoni G, Lomivorotov VV, Alvaro G, Lobreglio R, Pisano A, et al. (2017) Levosimendan for Hemodynamic Support after Cardiac Surgery. N Engl J Med 376 .

4. Caruba T, Hourton D, Sabatier B, Rousseau D, Tibi A, et al. (2016) Rationale and design of the multicenter randomized trial investigating the effects of levosimendan pretreatment in patients with low ejection fraction $(\leq 40 \%)$ undergoing CABG with cardiopulmonary bypass (LICORN study). J Cardiothoracic Surg 11: 127.

5. Harrison RW, Hasselblad V, Mehta RH, Levin R, Harrington RA, et al. (2013) Effect of levosimendan on survival and adverse events after cardiac surgery: a meta-analysis. J Cardiothoracic Vascular Anesth 27: 1224-32.

6. Toller W, Heringlake M, Guarracino F, Algotsson L, Alvarez J, et al. (2015) Preoperative and perioperative use of levosimendan in cardiac surgery: European expert opinion. Int J Cardiol 184: 323-36.

7. Gordon AC, Perkins GD, Singer M, McAuley DF, Orme RM, et al. (2016) Levosimendan for the prevention of acute organ dysfunction in sepsis. $\mathrm{N}$ Engl J Med 375: 1638-48.

8. Cleland JG, Takala A, Apajasalo M, Zethraeus N, Kobelt G (2003) Intravenous levosimendan treatment is cost-effective compared with dobutamine in severe low-output heart failure: an analysis based on the international LIDO trial. Eur J Heart Fail 5: 101-8.

9. Fedele F, D'ambrosi A, Bruno N, Caira C, Brasolin B, et al. (2011) Costeffectiveness of levosimendan in patients with acute heart failure. J Cardiovascular Pharmacol 58: 363-6.

10. Lucioni C, D’Ambrosi A, Mazzi S, Pollesello P, Apajasalo M, et al. (2012) Economic evaluation of levosimendan versus dobutamine for the treatment of acute heart failure in Italy. Adv Ther 29: 1037-50.

11. Mardiguian S, Kivikko M, Heringlake M, Smare C, Bertranou E, et al. (2016) Cost-benefits of incorporating levosimendan into cardiac surgery practice: German base case. J Med Econ 19: 506-14

12. Nieminen MS, Buerke M, Parissis J, Ben-Gal T, Pollesello P, et al. (2015) Pharmaco-economics of levosimendan in cardiology: A European perspective. Int J Cardiol 199: 337-41. 